\title{
Minimal Residual Disease-Based Risk Stratification in Acute Leukemia
}

National Cancer Institute

\section{Source}

National Cancer Institute. Minimal Residual Disease-Based Risk Stratification in Acute

Leukemia. NCI Thesaurus. Code C122456.

Risk stratification of acute lymphoblastic leukemia, and less often acute myeloid leukemia, based on minimal residual disease levels. 